\title{
Dripping dynamics of Newtonian liquids from a tilted nozzle
}

\author{
Amaraja Taur ${ }^{\mathrm{a}}$, Pankaj Doshi ${ }^{\mathrm{b}}$, Hak Koon Yeoh ${ }^{\mathrm{a}, *}$ \\ a Department of Chemical Engineering, Faculty of Engineering, University of Malaya, 50603, Kuala Lumpur, Malaysia \\ ${ }^{\mathrm{b}}$ Chemical Engineering and Process Development Division, National Chemical Laboratory, 411008, Pune, India
}

H I G H L I G H T S

- Dripping regimes from an inclined nozzle are mapped.

- Correlation for drop volume is reported over wide ranges of parameters.

- Results suggest significant weakening of capillary forces by asymmetry.

A R T I C L E I N F O

\section{Article history:}

Received 18 June 2014

Received in revised form

8 December 2014

Accepted 15 December 2014

Available online 24 December 2014

Keywords:

Asymmetric dripping

Inclined nozzle

Breakup time

Drop volume

Phase diagram

\begin{abstract}
A B S T R A C T
The dripping dynamics of Newtonian liquids emanating from an inclined nozzle is studied. The fluid viscosity $\mu$, flow rate $Q$, nozzle radius $R$, and inclination angle $\theta$ have been varied independently. The drop breakup times and the different modes of dripping have been identified using high speed imaging. A phase diagram showing the transition between the dripping modes for different $\theta$ is constructed in the (We, Ka) space, where We (Weber number) measures the relative importance of inertia to surface tension force and $\mathrm{Ka}$ (Kapitza number) measures the relative importance of viscous to surface tension forces. At low values of $W e$ and $K a$, the system shows a transition from period-1 to limit cycle before chaos. The limit cycle region narrows down with increase in inclination. Further increase in the values of We and $K a$ gives a direct transition from period- 1 to chaos. The new experiments reveal that in the period- 1 region, increasing the nozzle inclination angle $\theta$ results in lowering of the drop breakup time $t_{b}$, suggesting that the surface tension forces cannot hold the drops longer despite the weakened effective gravitational pull. This counter-intuitive finding is further corroborated by pendant drop experiments and computations. More curiously, throughout the period-1 regime, the drop volume is independent of the flow rate. This resulted in a relatively simple correlation for the dimensionless drop volume $\mathrm{V}=1.3 \mathrm{G}^{-1} \mathrm{Ka}^{0.02}(\cos \theta)^{0.37}$ accurate to within $10 \%$ over wide ranges of the independent variables.
\end{abstract}

(C) 2014 Elsevier Masson SAS. All rights reserved.

\section{Introduction}

Drop formation from a vertical nozzle finds many industrial applications such as ink-jet printing [1], silicon microstructure for protein identification [2], microencapsulation [3], and 3D printing [4]. Quite a number of these processes are operated under a constant flow rate. This process shows a variety of interesting behaviour. Dripping mode is characterized by tiny droplets emanating from the nozzle at low flow rates [5-8], while jetting mode occurs at high flow rates in which liquid flows out as a continuous stream to form a jet which subsequently breaks up into smal droplets $[9,10]$. Such behaviour has been studied for centuries. Eggers [11] provided an extensive review about the experimental and

\footnotetext{
* Corresponding author. Tel.: +60379675360. E-mail address: yeohakoon@um.edu.my (H.K. Yeoh).
}

computational work starting from 1686. Due to the encyclopedic amount of prior work, in the following, only key ideas and references pertinent to this work will be covered.

The most commonly investigated configuration is a Newtonian liquid having viscosity $\mu$, density $\rho$, and surface tension $\sigma$, flowing through a nozzle of outer radius $R$, at flow rate $Q$. For a vertical nozzle the dripping dynamics are governed by three dimensionless groups [12-14]: the Weber number We $=\rho v^{2} R / \sigma$, where $v$ is the velocity of the emanating liquid, that measures the relative importance of inertial to surface tension force, the Bond number $G=\rho g R^{2} / \sigma$, where $g$ is the acceleration due to gravity, that measures the relative importance of body force to surface tension force, and the Kapitza number Ka $=\left(\mu^{4} \mathrm{~g} / \rho \sigma^{3}\right)^{1 / 3}$ or the Ohnesorge number $\mathrm{Oh}=\mu /(\rho R \sigma)^{1 / 2}$, both measuring the relative importance of viscous force to the surface tension force $[13,15]$. The former is more convenient for studies on macroscopic dynamics as it is independent of the characteristic length scale of the system, 
whereas the latter is the preferred group when the focus is on the dynamics of the pinch-off singularity in drop breakup [11,16].

The different modes of dripping seen in experiments include period-1 dripping where every drop is of equal size, period- $n$ ( $n=$ $2,3,4, \ldots$ ) dripping where every $n$-th drop is identical, and higher odd-period or chaotic mode of dripping [12-14]. The increasing complex dripping behaviour occurs at increasing flow rates (or $W e$ ). At very low flow rates, a tiny satellite drop often follows the primary drop. At high viscosities (high $\mathrm{Oh}$ ), the dripping behaviour simplifies to either P1 with satellites or jetting [13]. A detailed phase diagram showing transitions from complex to simple dripping and jetting in the (We, Oh) space had been reported [13]. The critical We for the transitions to jetting were estimated by scaling arguments and shown to accord well with simulations [14]. Initially the phase diagram developed in $(\mathrm{We}, \mathrm{Oh})$ space was constructed for a moderate value of $G=0.5$ [14], but the response if the value of $G$ varies was unknown. This unexplored dripping dynamics for a wider range of $G$ was later studied by Subramani et al. [13]. It was found that at high values of $G$, the dripping dynamics is richer and tends to become chaotic at lower values of $W e$.

In addition to physical properties of the liquid, geometry also plays a critical role. Experimental results showed that the effect of nozzle thickness can be neglected within experimental error only when $R i / R>0.2$, where $R i$ is the inner radius of the nozzle [7]. For thin-wall nozzles, the dripping dynamics is on the whole the same. In addition, radical changes in the dripping dynamics were found when the nozzle shape changes from a flat tip to a bevelled shape [17]. Satellite drop formation is notably reduced in bevelledshape nozzles. In all previous studies the nozzle had a circular cross section. However changing the nozzle cross section to some other shapes is expected to give different droplet volumes as the capillary forces are changed by the shape of the liquid meniscus. For example, the study by Chen et al. showed that the volume of the droplet decreased by $18 \%$ when the nozzle cross section was changed from circular to triangular with a stretched corner [18].

The introduction of asymmetrical perturbations, both by tilting the nozzle at an angle $\theta$ with the vertical [19], and by cutting obliquely the tip of nozzle [20,21], breaks the cylindrical symmetry and induced strong changes in dripping dynamics. For an obliquely cut nozzle tip, the added degree of freedom enables transversal oscillations of a pendant drop, which interacted in a complicated manner with the other dynamics during breakup, giving shorter dripping times and more regular dripping pattern [20]. The bifurcation diagrams obtained by plotting the dripping time versus the flow rate for flat nozzle, bevelled nozzle, and obliquely cut nozzle showed different patterns. The frequency of drop oscillations decreased going from the flat nozzle tip to the bevelled nozzle tip and to the obliquely cut nozzle [21]. It was claimed that this was due to wetting characteristics of the liquid with the wall of the nozzle as it determines three phase contact lines affecting the dripping time series behaviour [21]. In the experiments on dripping from a tilted nozzle, the measured time $\left(T_{n}\right)$ between the $n$th and $(n+1)$ th drops were plotted, giving time return maps for different nozzle inclination angle $\theta$. The results showed strong changes in the attractor topology, suggesting that inclination angle can be an effective control parameter for the dripping dynamics [19].

Despite the rich dynamics of dripping from a tilted nozzle, we failed to uncover any other articles in the English literature. Due to the limited range of parameters studied previously [19], the more general behaviour of dripping from a tilted nozzle remains unknown. Compounding the difficulty, the presence of asymmetry prevented simple theoretical analysis. Rigorous numerical studies of the effects of asymmetry began with the more modest, albeit pioneering studies on the static shape and the stability of a pendant or sessile drop [22,23]. Unfortunately, as recently as in 2012 [24], the simulation efforts did not seem to have advanced ultimately into dripping dynamics. One very likely obstacle is the scarcity of openly available experimental data to validate the simulations. To assist in this endeavour, the main goal of this paper is to develop a comprehensive picture of the dripping dynamics from a tilted nozzle. In order to achieve that goal, (a) dripping dynamics will be explored through the study of the breakup time $\tilde{t}_{b}$, which is the time interval between two subsequent drop breakups, and (b) dripping phase diagrams for different values of $\theta$ will be constructed.

The paper is organized as follows: Section 2 describes the material properties, experimental setup, and experimental procedure with data analysis methods for studying drop formation from both a vertical and an inclined nozzle. Section 3 details the experimental results and key findings based on breakup times and their comparison at different values of $\theta$. Section 4 concludes this work.

\section{Experiments}

\subsection{Apparatus}

The experimental apparatus is depicted in Fig. 1. It consists of a nozzle through which liquid flows to form drops. The liquid was delivered to the nozzle by using a Meditech/ZB-1800D Syringe Pump which is capable of providing range of flow rate from 0.00167 to $30 \mathrm{~mL} / \mathrm{min}$ with an accuracy of $\pm 2 \%$. Stainless steel dispensing nozzles (P-30619-06, P-30619-01, and P-30619-07) were obtained from Cole-Parmer. The outer radii of the nozzles are $0.625 \mathrm{~mm}(\mathrm{~N} 1), 0.100 \mathrm{~mm}(\mathrm{~N} 2)$, and $1.96 \mathrm{~mm}(\mathrm{~N} 3)$. The ratio of the inner radius to outer radius is $<0.2$, hence the effect of nozzle thickness on the interface dynamics can be safely neglected [7]. A protractor is provided to adjust the tilt angle of the nozzle. A transparent shield is provided to reduce draught that can perturb the drop formation process.

The high speed camera is Casio EX-FH100 capable of recording 30-1000 frames per second. A planar white LED backlight measuring $10 \mathrm{~cm} \times 10 \mathrm{~cm}$ (model LFL-Si100-W-IP65) with adjustable brightness was obtained from Falcon Illumination (M) Pte. Ltd. The sharpness of the images can be adjusted via the intensity of the backlight, the focal length and digital zoom of the camera. All parts of apparatus except the syringe pump were kept on a $0.3 \mathrm{~m} \times 0.3 \mathrm{~m} \times 0.06 \mathrm{~m}$ aluminium optical base plate inside the transparent shield.

\subsection{Fluid characterization}

Mixtures of water and glycerol were chosen because their surface tension and densities are close to that of pure water, but their viscosities can be made to vary by three orders of magnitude. The $99 \%$ pure glycerol was obtained from $\mathrm{R}$ and $\mathrm{M}$ Chemicals, CAS NO [56-81-5], and used as obtained. Distilled water was used to the make water-glycerol mixtures. The physical properties of these water-glycerol mixtures are taken from the literature [25] and are listed in Table 1. Silicone oil (Dow Corning ${ }^{\circledR} 111$ Valve Lubricant and Sealant) was used to prevent wetting of the outer nozzle surface.

The image captured by using just water-glycerol mixtures showed a colour gradient within the drop area, which was inconvenient for the subsequent automated image analysis. To minimize the colour gradient within the drop area, methylene blue dye was added in the water-glycerol mixture. Analysing the images for the dyed solution showed that $0.5 \mathrm{wt} \%$ was the minimum required. The surface tensions of the dyed water-glycerol solutions were measured using Langmuir Blodgett trough from KSV instruments. The viscosity measurements were carried using an ARES rheometer equipped with the cup and bob geometry. The liquid densities were 


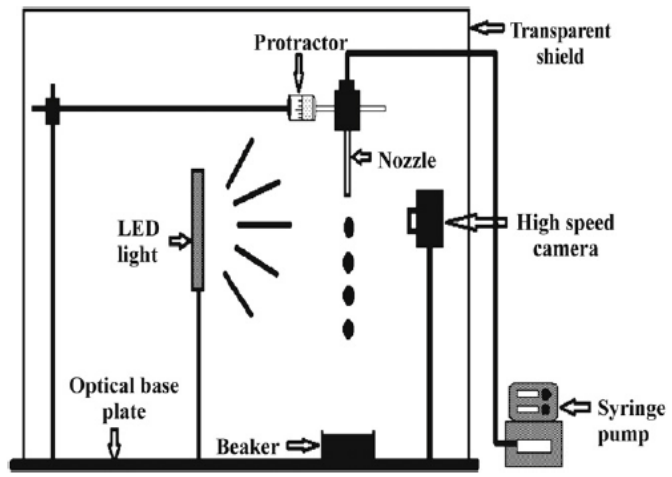

Fig. 1. Schematics of the experimental setup.

Table 1

Physical properties of water-glycerol mixtures [25].

\begin{tabular}{lllll}
\hline Solution & Wt. \% glycerol & $\rho\left(\mathrm{kg} / \mathrm{m}^{3}\right)$ & $\mu(\mathrm{mPas})$ & $\sigma(\mathrm{mN} / \mathrm{m})$ \\
\hline S0 & 0 & 1000 & 1 & 72 \\
S20 & 20.0 & 1044 & 1.5 & 69.5 \\
S40 & 40.0 & 1095 & 3.2 & 68.4 \\
S80 & 80.0 & 1205 & 45.9 & 64.7
\end{tabular}

measured using the known volume of liquid using a weighing balance, AND model GF-300. All the physical property measurements for the dyed water-glycerol mixtures were performed at $25 \pm 1{ }^{\circ} \mathrm{C}$. The measurement errors for density, surface tension and viscosity were within $\pm 1 \%$ accuracy. These measurements showed that the effect of dye on the physical properties of the water-glycerol solutions could be safely neglected within experimental error.

\subsection{Experimental procedure}

The experimental run began with applying a thin layer of silicone oil on the outer wall of the nozzle. This pinned the liquid to the outer sharp edge of the nozzle tip even at inclined positions. The verticality of the nozzle was checked with two perpendicularly mounted pendulums. Following this, the tilt angle was adjusted using the protractor. The prepared solution was drawn into the syringe and any bubble present in the syringe or tube was purged off. A desired flow rate was then set on the syringe pump. To minimize the influence of start-up transients, at least 50 drops of liquid were formed prior to recording. Preliminary tests using

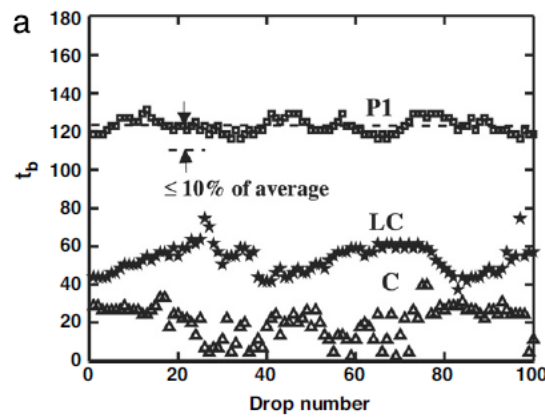

different waiting periods prior to recording affirmed that this was sufficient. The drop formation sequences were recorded at 240-420 frames per second depending upon the liquid flow rate. The recorded videos were first converted into images with the Image software [26], then analysed using an algorithm written in MATLAB ${ }^{\text {TM }}$ software to detect the drop breakup and to calculate the breakup time $\tilde{t}_{b}$ between two successive drops.

In this work, the experiments were performed at different values of We $\left(5 \times 10^{-4}\right.$ to 0.45$)$ by varying the liquid flow rate $Q, K a\left(3.22 \times 10^{-4}\right.$ to 0.0526$)$ by changing the viscosity $\mu$ of the liquid, $G(0.053-0.70)$ by using three different nozzle radius $R$, and $\theta\left(0^{\circ}, 30^{\circ}\right.$, and $\left.60^{\circ}\right)$. All the experiments were carried out at room temperature $\left(25 \pm 1{ }^{\circ} \mathrm{C}\right)$.

\section{Results and discussions}

\subsection{Dripping modes}

Experiments with a vertically oriented nozzle illustrate the various dripping modes observed. The breakup time, $\tilde{t}_{b}$ was made dimensionless using capillary time $\sqrt{\rho R^{3} / \sigma}$. Throughout the remaining part of this paper, a variable with a tilde designates a dimensional variable while one without denotes the dimensionless counterpart of the same variable. Fig. 2 shows the variation of the dimensionless breakup time $t_{b}$, with drop numbers. As depicted in Fig. 2(a), values of $t_{b}$ decreases with increase in Weber number. Based on this, three different dripping regimes were encountered, namely period-1 (P1), limit cycle (LC), and chaos (C). At low Weber numbers, every droplet had the same $t_{b}$ value, and this mode was denoted as the period-1 dripping (P1). Seen on the time return map in Fig. 2(b), the points cluster together, ideally forming only one point. At moderate Weber numbers, the $t_{b}$ trajectory repeated itself, showing low amplitude and low frequency oscillations around the average. This was denoted as the limit cycle (LC) behaviour. On the time return map, the trajectory encircles a region. The chaotic (C) behaviour was seen at high Weber numbers, where the $t_{b}$ trajectory did not repeat itself, showing disorderly long term evolution. On the time return map, the points are scattered rather randomly. Raising the angle of tilt to $\theta=60^{\circ}$ at constant $W e, K a$, and $G$, the evolution of $t_{b}$ with drop number and the time return map is shown in Fig. 3. The features remain qualitatively identical. This is notable as the extent of asymmetry in the liquid meniscus is significant at this inclination. It suggests that inclination probably exerts higher order effects on the dripping dynamics. Nevertheless, comparing Figs. 2 and 3, the values of $t_{b}$ for the P1 regime decrease noticeably with an increase in $\theta$, whereas the values for $t_{b}$ for the LC and the $C$ modes of dripping are relatively independent of $\theta$. Since

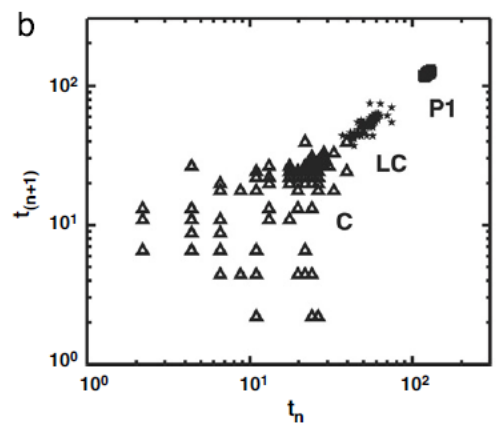

Fig. 2. (a) Variation of the dimensionless dripping time with drop number and (b) the corresponding time return map for a vertical nozzle. Three different dripping behaviours

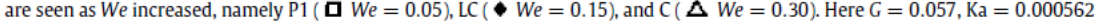



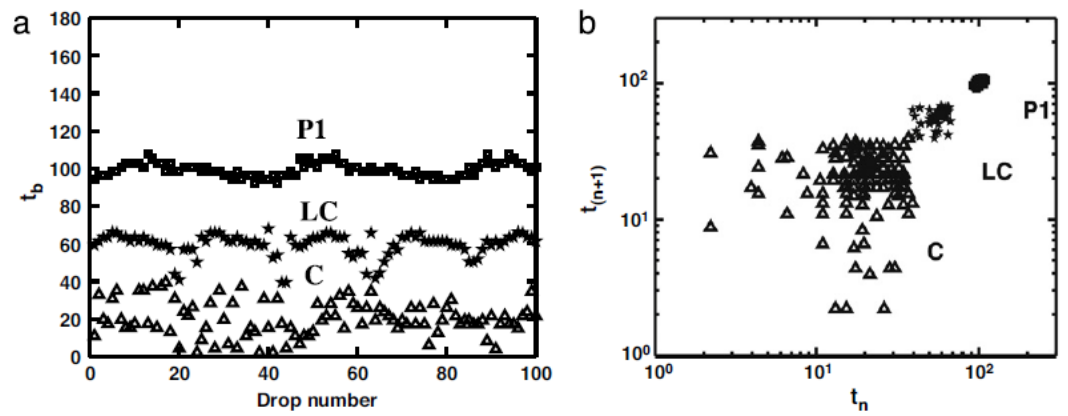

Fig. 3. (a) Variation of the dimensionless dripping time with drop number and (b) the corresponding time return map for $\theta=60^{\circ}$. Three different dripping behaviours are seen as We increased, namely P1 $(\boldsymbol{\square} W e=0.05), \mathrm{LC}(\bullet e=0.15)$, and $\mathrm{C}(\Delta W e=0.30)$. Here $G=0.057, \mathrm{Ka}=0.000562$.
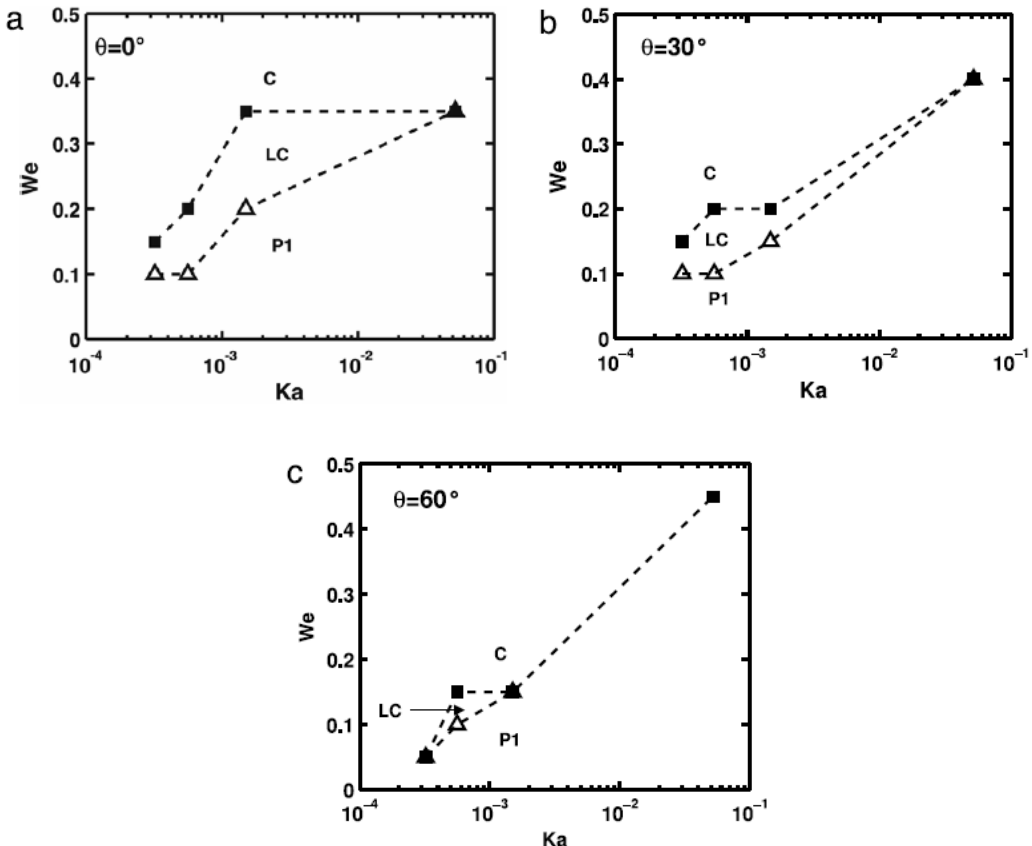

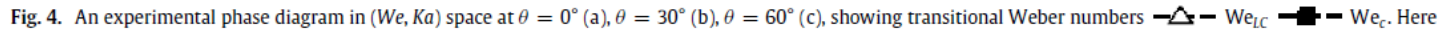
$G=0.062$

the flow rate remains identical, the droplets formed from the inclined nozzle in the P1 regime must be smaller than their counterparts in the vertical case. This opens up another avenue to regulate drop volumes.

To facilitate discussions, the transition from P1 to LC is deemed to occur at $W e=W e_{L C}$, and the transition from LC to C occurs at $W e=W e_{c}$. These two Weber numbers can be pin-pointed experimentally. As the value of $\mathrm{Ka}$ changes, the corresponding values of $W e_{L C}$ and $W e_{c}$ also change. The loci of these transitional We are plotted against $\mathrm{Ka}$ as a dripping mode phase diagram next.

\subsection{Phase diagram}

The phase diagrams shown in Fig. 4(a)-(c) identify the location in the parameter space where the dynamics changes from one

mode to another. For a vertical nozzle (Fig. 4(a)), at low values of $K a$, both $W e_{L C}$ and $W e_{c}$ rise sharply as the value of $K a$ increases. For high values of $K a$, the trajectories of $W e_{L C}$ and $W e_{c}$ converge, i.e. the transition occurs directly from P1 to $\mathrm{C}$ without exhibiting a LC regime. This is known as the "simple dripping" region in the computational phase diagram of Subramani et al. [13] when $G=0.3$. Noting that $K a=\left(O h^{4} G\right)^{1 / 3}$, their corresponding value of $K a$ for such "simple dripping" regime is 0.67 , nearly an order of magnitude larger than ours. Further, in their work, the P1 region narrows down prior to the "simple dripping" regime, whereas ours do not exhibit this narrowing. Certainly, keeping the P1 region wide at high values of $K a$ avails greater flexibility for applications. These differences in the phase diagram highlight the very considerable effect of the value of $G$ on the dripping dynamics, also illustrated by the same authors.

\section{Link to Full-Text Articles :}

\section{http://www.sciencedirect.com/science/article/pii/S0997754614001939}

